\title{
Continuous Positive Airway Pressure as a Potential Treatment for Preeclampsia
}

\author{
Visasiri Tantrakul ${ }^{1}$, Jee Hyun $\mathrm{Kim}^{2}$ and Christian Guilleminault $\mathrm{l}^{3, *}$ \\ ${ }^{1}$ Sleep Disorder Center and Pulmonary and Critical Care, Ramathibodi Hospital, Mahidol University, Bangkok, \\ Thailand \\ ${ }^{2}$ Dankook University College of Medicine, Thailand \\ ${ }^{3}$ Sleep Medicine Division, Stanford University, Redwood City, CA, Thailand
}

\begin{abstract}
Accumulating data indicate that obstructive sleep apnea is an independent risk factor for gestational hypertension and preeclampsia, contributing to adverse maternal and fetal outcomes. Preliminary studies have demonstrated the benefits of early nasal continuous positive airway pressure (CPAP) treatment in pregnant women who are at high risk for preeclampsiato prevent pre-eclampsia and adverse maternal and fetal outcomes. High-risk pregnant women - such as those with obesity, chronic hypertension, and a prior history of preeclampsia- should be evaluated for diagnosis and treatment of sleep-disordered breathing given the possible benefit of early CPAP application. The pathophysiological evidence linking preeclampsia and sleep-disordered breathing was also reviewed to support the rationale for CPAP application in addition to conventional preeclampsia treatment. Improved knowledge among healthcare providers about sleep disorders during pregnancy is needed, and large scale studies should be performed to investigate the role of early CPAP intervention during pregnancy.
\end{abstract}

Keywords: Preeclampsia, Sleep-disordered breathing, Nasal CPAP, Outcomes.

\section{INTRODUCTION}

Hypertensive disorders in pregnancy are the most common medical complications of pregnancy. These can be divided into three categories: chronic hypertension, gestational hypertension (GH), and preeclampsia. Preeclampsia is the major cause of maternal and fetal morbidity and mortality worldwide, affecting $3-5 \%$ of all pregnancies [1]. It is associated with a perinatal and neonatal mortality rate of $10 \%$ [2]. It is characterized by the new onset of hypertension with a resting blood pressure at least $140 / 90 \mathrm{mmHg}$, proteinuria (24-hour urine protein $>0.3 \mathrm{~g}$ ), and edema occurring after 20 weeks of gestation [3]. The key pathogenesis of preeclampsia occurs with abnormal placentation, followed by secretion of toxic placental factors that induce widespread injury to the maternal vascular endothelium, leading to dysfunction of the kidneys, liver, brain, and blood coagulation system [4]. Risk factors for preeclampsia include: primiparity, multifetal gestation, extremes of maternal age, and a previous history of preeclampsia, obesity, hypercoagulable state, chronic hypertension, and diabetes [2, 5-7]. Moreover, there is growing evidence demonstrating an association between sleep-disordered breathing (SDB) and preeclampsia with adverse feto-maternal outcomes.

*Address correspondence to this author at the Sleep Medicine Division, Stanford University, 450 Broadway Street, Redwood City, CA 94063, Thailand; E-mail: cguil@stanford.edu
Pregnancy and sleep have a complex interaction. Sleep quality and duration are commonly affected during pregnancy. Changes in sleep patterns are due to several hormonal, physiological, physical, and behavioral factors which exist during pregnancy. The physiologic and biochemical changes of pregnancy may place women at risk for developing specific sleep disorders such as obstructive sleep apnea and restless legs syndrome. The pregnancy related changes might also exacerbate or unmask preexisting sleep disorders, in particular SDB.

In this review, we discuss sleep changes and SDB during pregnancy, especially in relation to preeclampsia. This review also describes the reported association between SDB and adverse pregnancy outcomes. We also suggest treatment for preeclampsia that exceeds the standard convention, in light of the effects on medical status and fetal outcome.

\section{SLEEP CHANGES DURING PREGNANCY AND PREECLAMPSIA}

As pregnancy progresses, hormonal and physical changes cause alterations in sleep duration and architecture. While total sleep time increases during the first trimester compared to the non-pregnant period, thereafter it progressively decreases and is significantly reduced during the third trimester. Deep sleep (NREM stage 3 and 4) and REM sleep are reduced after the first trimester [7-9].

In contrast to data obtained during normal pregnancy, Edwards et al. reported marked alterations in sleep architecture in 25 pre-eclamptic women compared to 17 normal pregnant women with a substantial increase in the 
percentage of slow wave sleep $(43 \pm 3$ versus $21 \pm 2 \%, \mathrm{p}<$ $0.001)$. The authors postulated that cerebral edema and cytokine release might be the possible explanations for the increase in slow-wave sleep [10]. Tumor necrosis factoralpha (TNF-alpha), interleukin-6 (IL-6), and IL-8 have all been identified in preeclampsia $[11,12]$. Some of the cytokines including IL-1, TNF-alpha, and prostaglandins have been suggested as factors that increase slow-wave sleep [13]. The report also mentions a decrease in the percentage of REM sleep and an increase in REM latency, although these changes were most likely due to the clonidine that a majority of the preeclamptic subjects had been taking in this study [10].

\section{SLEEP-DISORDERED BREATHING DURING PREGNANCY}

Pregnancy causes anatomic, physiologic, and hormonal changes and a narrowing of the size of the upper airway which may increase the risk for developing obstructive sleep apnea (OSA) or worsen pre-existing sleep apnea. Respiratory mechanics alter during pregnancy with a $20 \%$ decrease in the function residual capacity (FRC), which further decreases in the supine position and during sleep. Reduction in FRC results in decreased oxygenation in the mother from an increase in the alveolar-arterial gradient and an increase in ventilation-perfusion mismatch, whereas the increase in progesterone during pregnancy markedly up-regulates ventilatory drive $[14,15]$. Furthermore, during normal pregnancy there is a progressive rightward shift of the oxyhemoglobin dissociation curve which facilitates the offloading of oxygen to peripheral tissues, most importantly to the placenta. In contrast, in preeclampsia there is a leftward shift of the oxyhemoglobin dissociation curve which impairs oxygen off-loading and thereby renders the fetus vulnerable to even subtle obstructive respiratory events in the preeclamptic mother [16].

Snoring in pregnancy has been shown to occur most commonly in the third trimester. Loube et al. reported the prevalence of snoring in pregnancy using a questionnaire, comparing the results to non-pregnant women. The study showed $14 \%$ of pregnant women report snoring, which is about three times higher than non-pregnant women [17]. Franklin et al. suggested a relationship between habitual snoring and pregnancy-induced hypertension and preeclampsia [18]. Seven percent of pregnant women stated that they started to snore or markedly increased their snoring frequency during the first trimester, $6 \%$ during the second trimester, and $24 \%$ of during the third trimester.

Izci et al. reported a frequency of habitual snoring in healthy pregnant women during the third trimester of $59 \%$, compared to a frequency of $12 \%$ pre-pregnancy and a postpartum frequency of $18 \%$ [19]. However, the actual prevalence of OSA among pregnant women is unknown. Edwards et al. conducted a case-controlled, longitudinal study of SDB during late pregnancy (mean $32 \pm 4$ weeks of gestation) and postpartum ( mean $4 \pm 2$ months) in 10 women referred for suspected SDB during the third trimester of pregnancy [20]. They found that postnatal sleep apnea severity improved compared to late pregnancy in both apneahypopnea index (AHI) and minimum oxygen saturation in NREM (63 \pm 15 versus $18 \pm 4$ events per hour, $p=0.03)$ and in REM sleep $(64 \pm 11$ versus $22 \pm 4$ events per hour, $\mathrm{p}=$ 0.002). There was no relationship between the change in weight or BMI and the change in AHI from antenatal to postnatal sleep. Blood pressure response to sleep apnea peaked at $170-180 \mathrm{mmHg}$ antenatally, while it only reached a maximum of $130-140 \mathrm{mmHg}$ postnatally. This study indicates that late pregnancy may be associated with an increase in the severity of SDB and blood pressure response.

These findings convey an important message for antenatal care: If SDB is diagnosed prior to pregnancy, the condition needs to be reassessed (particularly at the third trimester of pregnancy), with treatment options discussed given the potential impact on the growing fetus. Moreover, even if SDB is not present prior to pregnancy, with risk factors such as obesity or craniofacial deficiency it is crucial to assess the patient during pregnancy to detect exacerbation of SDB. The severity of SDB should also be reassessed during the postpartum period.

\section{UPPER AIRWAY NARROWING}

During pregnancy, there are several potential physiological changes that predispose to an increase in upper airway resistance and reduce the cross-sectional area of the upper airway. These changes include: weight gain, a decrease in functional residual capacity (FRC) due to displacement of the diaphragm, pharyngeal edema of pregnancy, and the effects of sleep deprivation and fragmentation on pharyngeal dilator muscle activity and upper airway collapsibility $[14,15]$. Studies of upper airway dimension using acoustic reflection techniques in healthy pregnant women show that the upper airway is significantly narrower during the third trimester of pregnancy compared to non-pregnant women and during repeat measures during the postpartum period [19].

There are significant differences when comparing the reports of snoring in non-pregnant women (14\%), pregnant women $(28 \%)$, and in pregnant preeclamptic women $(75 \%)$ $(\mathrm{p}<0.02)$. Preeclamptic women also have narrower upper airway dimensions in both upright and supine position compared to non-pregnant women and women with an uncomplicated pregnancy. These changes in upper airway resistance may contribute to respiratory episodes during sleep in preeclamptic women, which could further increase their blood pressure [21].

Nasal patency is also reduced during pregnancy, [22-26] with $42 \%$ of women at 36 weeks gestation reporting nasal congestion and rhinitis [22]. Elevation in circulating estrogen concentrations and an increase in circulatory blood volume during pregnancy have been linked with nasal congestion.

\section{SLEEP DISORDERED BREATHING AND ADVERSE MATERNAL AND FETAL OUTCOMES.}

Sahin et al. studied the fetal outcomes in 4 pregnant women with OSA compared to 31 pregnant women without OSA and found that neonates of women with OSA had lower mean APGAR scores and lower birth weights than neonates of women without OSA [27]. Recent data from a large Taiwanese pregnancy cohort of 791 women with OSA versus 3955 women without OSA showed that having OSA increases the risk of low-birth weight (OR 1.76, 95\% CI 
1.28-2.4), preterm birth (OR 2.31, 95\% CI 1.77-3.01), small size for gestational age $(1.34,95 \%$ CI 1.09-1.66), cesarean section (OR 1.74, 95\% CI 1.48-2.04), and preeclampsia (OR $1.6,95 \%$ CI 2.16-11.26) [28]. This study is consistent with the prior results from Bourjeily et al. that evaluated 1000 pregnant subjects with a self-reported snoring rate of $35 \%$. This study shows that snoring increases the risk of having pregnancy-induced hypertension and preeclampsia (OR 2.3, 95\% CI 1.4-4.0), gestational diabetes (OR 2.1, 95\% CI 1.33.4), and unplanned Cesarean delivery (OR 2.1, 95\% CI 1.43.2) [29]. Screening for sleep-disordered breathing with the Berlin questionnaire (a questionnaire designed to recognize subjects at risk of OSA) was done in 4074 pregnant women presenting at delivery compared to 490 non-pregnant women presenting for outpatient surgery. Positive Berlin questionnaire were higher in the pregnant women compared to the non-pregnant women $(33 \%$ versus $20 \%, \mathrm{p}<0.001)$. The incidence of preeclampsia was greater (OR 3.9) in the pregnant women with a positive Berlin Questionnaire as compared to those with a negative Berlin questionnaire. However, the survey was performed during the third trimester at the time of delivery- and not in early pregnancy and therefore these results cannot be used for early prediction and intervention during the course of pregnancy [30]. Olivarez et al. [31] performed a prospective study screening for OSA in 100 pregnant women at 26-39 weeks of gestation using the Berlin questionnaire compared to polysomnography combined with fetal monitoring for $>3$ hours. In this study, the Berlin questionnaire showed poor predictive value for OSA (AHI $>5$ events/hour) in pregnancy with a sensitivity of $35 \%$ and a specificity of $63.8 \%$. Only the snoring component of the Berlin questionnaire correlated with oxygen desaturation $(<95 \%)$ $(p=0.003)$ and the sleepiness component failed to correlate with polysomnographic parameters [31]. Another report was consistent with prior studies, indicating that snoring and sleepiness increase during pregnancy both in normal pregnant and preeclamptic women. However, there was no correlation between sleepiness and snoring, potentially suggesting the presence of another cause of the sleepiness [32].

Worsening symptoms associated with SDB in pregnancy have been reported by Pien et al. These authors indicate that SDB symptoms (based on the apnea symptom score from the Multivariate Apnea Prediction Index) and Epworth Sleepiness Scale increase significantlyfrom the first trimester to the month of delivery. This was not limited to snoring, but included gasping, choking, difficulty breathing, and apneic events. The increase in SDB symptoms compared to the first trimester was detectable by 28-29 weeks of gestation [33]. Despite these numerous reports, there are no specific guidelines to screen for SDB in pregnant women to date. Further research is needed to optimize the measures and strategy needed to screen for SDB in pregnancy.

\section{IMPACT OF SLEEP AND SLEEP-DISORDERED BREATHING ON PREECLAMPSIA}

During normal sleep, many hemodynamic changes occur, including reductions in cardiac output (CO) and total peripheral resistance (TPR) from wakefulness to NREM sleep and variability in these measures during REM sleep. Blyton et al. [34] studied hemodynamic changes during sleep in 24 women with severe preeclampsia compared to 15 controls with normal pregnancy. They found that during wakefulness hemodynamic parameters including stroke volume, heart rate, and $\mathrm{CO}$ were similar in controls and patients with preeclampsia but TPR was significantly elevated in the preeclampsia group. During sleep, heart rate, stroke volume, and cardiac output decreased in preeclamptic subjects with a substantial increase in TPR and blood pressure. Relative changes in hemodynamics during normal pregnancy were similar to those seen in non-pregnant subjects, while women with preeclampsia had markedly altered responses during sleep. Most importantly, these women had reductions in $\mathrm{CO}$ associated with increases in TPR and blood pressure. These authors also found that $\mathrm{CO}$ during sleep was associated with fetal birth weight ( $\mathrm{r} 2=0.64$, $\mathrm{p}<0.001)$. Randomization to nasal CPAP, an intervention provided to half of the preeclamptic subjects, was also performed in this study. The results indicate that after CPAP treatment, these hemodynamic changes were minimized with a reduction in blood pressure during sleep. Interestingly, blood pressure and TPR were maximal during SWS sleepin preeclamptic subjects. This may result from increased upper airway resistance in the absence of arousal often found in these sleep stages leading to hypercapnia and an increase in sympathetic nervous system activity. Moreover, $\mathrm{CO}$ was at its nadir during stage 3 and 4 NREM sleep, demonstrating that slow-wave sleep is the time of greatest cardiovascular vulnerability in preeclampsia [34].

Various hypotheses have been proposed regarding the relationship between snoring and partial airway obstruction and an increase in nocturnal blood pressure. Previous studies have shown that snoring and OSA are associated with specifically nocturnal hypertension and "flattening" of the diurnal blood pressure rhythm (i.e. an absence of normal "dipping" of the blood pressure curve during the nocturnal period). Hypertension associated with preeclampsia usually demonstrates diurnal flattening or even a reversal of the nocturnal blood pressure, becoming increased relative to daytime blood pressure [35-37]. It has been shown that pregnancies complicated by preeclampsia are characterized by an increase in systolic blood pressure (BP) by $95 \%$ and diastolic BP by $13 \%$ between mid-gestation and delivery. Additionally, systolic (but not diastolic) BP may be slightly elevated during the first half of pregnancy in women who develop preeclampsia compared to those with gestational hypertension [38]. The circadian pattern of BP may also be a valuable disease predictor as significant changes in the mean of both systolic and diastolic BP, as adjusted for circadian rhythm, and elevation in the 24-hour mean pulse pressure have been reported in women who develop preeclampsia [39, 40].

Edwards et al. studied 11 preeclamptic women on 2 consecutive polygraphic sleep studies with simultaneous beat-to-beat blood pressure monitoring. Sleep-induced partial airflow limitations were present in all subjects during the first night of study and these flow limitations were subsequently eliminated with an autoset nasal CPAP with a maximum pressure of $6 \pm 1 \mathrm{cmH} 2 \mathrm{O}$ during the second night. Blood pressure was markedly reduced on the treatment night $(128 \pm 3 / 73 \pm 3)$ as compared to the first study night $(146 \pm 6 / 92 \pm 4)$. These authors concluded that partial airway obstruction in preeclampsia is associated with 
an increase in blood pressure, which can be eliminated with nocturnal CPAP use [41]. Preeclamptic patients with coexisting obstructive sleep apnea (OSA) show more augmented hemodynamic responses than normal pregnant women with OSA. Blood pressure responses to obstructive respiratory events during sleep were markedly augmented in preeclamptic patients compared with normotensive patients both in NREM $(38 / 24 \mathrm{mmHg}$ vs.20/12mmHg) and REM sleep $(46 / 28 \mathrm{mmHg}$ vs.24/13mmHg), despite similarities in the severity of upper airway obstruction during sleep. [42] This finding has important implications for the monitoring and treatment of preeclampsia. Fluctuations in BP resulting from coexisting OSA or an increase in upper airway resistance during sleep in preeclamptics may alter blood pressure control and further increase the risk of fetal complication. This is detrimental to preeclamptic women since data demonstrate a higher frequency of snoring and a more narrow upper airway dimension compared to normal pregnant women.

Connolly et al. reported a study of airflow limitation during sleep studies in 15 preeclamptic women compared to 15 pregnant women in each trimester and 15 non-pregnant women [43]. Although all subject groups had no significant OSA (AHI $<5$ events/hour), preeclamptic women spent more time with airflow limitation ( $31 \pm 8.4 \%$ of sleep period time) compared to normal pregnant subjects in their third trimester of pregnancy $(15.5 \pm 2.3 \%$ of sleep period time) and $<5 \%$ in the other three groups $(p=0.001)$. The pattern of flow limitation in preeclamptic women consisted predominately of prolonged episodes lasting several minutes without associated oxygen desaturation. These episodes were in contrast to shorter transient episodes of flow limitation or sequences of breaths with increased respiratory effort terminated by arousal, which is currently scored as respiratory effort related arousal (RERA), a feature of upper airway resistance syndrome (UARS) [44, 45]. This type of prolonged breathing pattern has been reported to occur during slow-wave sleep, which increases in preeclampsia. In sum, this study emphasized that inspiratory flow limitation is common during sleep in preeclampsia despite the absence of overt OSA with the conventional classification of severity of sleep apnea.

Using overnight polysomnography, Reid et al recently reported a high prevalence of sleep-disordered breathing in 34 pregnant women with gestational hypertension compared to 26 healthy pregnant controls [46]. There were significantly higher frequencies of SDB (respiratory disturbance index, RDI $>5$ per hour) in women with gestational hypertension than in uncomplicated pregnant women $(53 \%$ versus $12 \%, \mathrm{p}<0.001)$, although women with gestational hypertension were more obese. Interestingly, the selected cut-off point for SDB was very sensitive, with the rationale that even mild SDB had been shown to impact hemodynamics in a non-pregnant population. Also noted in this study, REM sleep was associated with both higher RDI $(28.1 \pm 39.4$ versus $5.9 \pm 9.8, \mathrm{p}=0.006)$ and oxygen desaturation index $>3 \%(9.4 \pm 15.7$ versus $1.1 \pm 4.5, \mathrm{P}=$ 0.02 ) in women with gestational hypertension, raising the concern of profound cardiovascular consequences of obstructive respiratory events given the higher sympathetic nervous activity in REM sleep. In a prior case-control polysomnographic study by Champagne et al. involving 17 pregnant women with gestational hypertension versus 33 pregnant women without hypertension, the finding of OSA (with AHI greater than 15 events per hour) was positive in 14 out of $17(82 \%)$ and in 15 out of $33(45 \%)$, respectively. The mean AHI was $38.6 \pm 36.7$ versus $18.2 \pm 12.2$ events/hour [47]. The adjusted odd ratio of OSA given the presence of gestational hypertension was $7.5 \quad(95 \%$ confidence interval 3.5-16.2). Based on these two studies, the prevalence of SDB and OSA is high among women with gestational hypertension despite using different cut-off points.

Data suggest the possible relationship between disordered breathing during sleep and preeclampsia, whether this is due to an increase in upper airway resistance or inspiratory airflow limitation during sleep. However, the question of whether sleep-disordered breathing is a causative factor or a consequence of preeclampsia due to generalized edema remains unaddressed. The above findings have important implications for the pathophysiology and the treatment of preeclampsia with CPAP. This is particularly true in light of the knowledge that in an earlier study in nonpregnant subjects Guilleminault et al indicated that, even in the absence of classic apnea or hypopnea or significant drops in oxygen saturation $(<90 \%)$, repetitive increases in blood pressure can occur as a result of upper airway resistance during sleep. These authors emphasized that in some patients with UARS and borderline hypertension, blood pressure can be controlled with treatment with CPAP [48].

\section{POTENTIAL PATHOPHYSIOLOGIC MECHANISM FOR PREECLAMPSIA IN RELATION TO SDB}

Despite growing evidence indicating an association between snoring and sleep apnea as potential risk factors for gestational hypertension and preeclampsia, direct evidence to support the potential pathophysiological mechanism linking these two conditions remains sparse [49].

Pathologic studies in preeclampsia show the abnormal development of an ischemic placenta, producing toxic placental factors which induce widespread endothelial dysfunction resulting in an imbalance of vasoactive mediators leading to vasoconstriction. An imbalance in circulating angiogenic factors is emerging as a prominent mechanism that mediates endothelial dysfunction. However, the initial triggering etiologic agents are currently unknown $[50,51]$. It is noteworthy that the general risk factors associated with severe preeclampsia significantly overlap with those reported to increase the risk of sleep-disordered breathing syndromes.

Sleep apnea is also associated with increased sympathetic activity with vasospasm, endothelial dysfunction, inflammation, and increased angiotensin II levels, findings that are also implicated in the pathogenesis of preeclampsia [50]. A study by Bachour et al. indicates that preeclamptic women have significantly more nasal flow limitation during sleep, higher interleukin-6 and tumor necrotic factor alpha plasma levels, more edema, and worse pregnancy outcomes than healthy pregnant women [52]. Another study supporting the association of preeclampsia with sleep-disordered breathing and endothelial dysfunction was performed using ambulatory sleep monitoring (Watch-PAT100) and noninvasive evaluation of endothelial function with a 
reactive hyperemia test (Endo PAT 2000). A higher ratio of post-to pre-occlusion pulse wave amplitude (endothelial function index, EFI) indicated better endothelial function. The results reveal that preeclamptic women have a significantly higher respiratory disturbance index (RDI) and a lower EFI compared to controls. Blood pressure correlates with RDI and EFI. The authors speculated that respiratory disturbances might contribute to the functional abnormality of blood vessels in preeclampsia [53].

\section{CPAP AS A POTENTIAL TREATMENT IN PREECLAMPSIA}

Nasal continuous positive airway pressure (CPAP) has been proposed as therapy for preeclampsia. A pilot prospective, longitudinal study in 12 pregnant women with preeclampsia risk factors was performed by Guilleminault $e t$ al to characterize SDB patterns and describe the effects of early nasal CPAP therapy [54]. The study included 2 subjects with a history of prior preeclampsia, 7 subjects had chronic hypertension without prior preeclampsia, and 3 were obese subjects. At initial evaluation, during the first trimester (mean 7.5 weeks of gestation), all women had mild sleepdisordered breathing with a mean respiratory disturbance index of $8.5 \pm 2.6$ events/hour. All subjects were compliant to nightly CPAP treatment throughout pregnancy with the use of heated humidification and the pressure set to 5-6 $\mathrm{cmH} 2 \mathrm{O}$ at initial titration, but subsequently requiring higher pressures of 8-9 $\mathrm{cmH} 2 \mathrm{O}$ when reaching the sixth month of pregnancy. All 7 women with chronic hypertension were able to maintain their blood pressure $<140 / 90 \mathrm{mmHg}$ during pregnancy without the need of medication adjustment and exhibited a normal nocturnal blood pressure with normal "dipping" related to the circadian rhythm. All women with pre-existing hypertension had normal deliveries of full-term infants with normal birth weights and APGAR scores [54]. However, pregnancy outcomes were worse in the 3 obese subjects. One subject had a miscarriage, 1 had a premature delivery at 34 weeks of gestation with the infant admitted to the neonatal intensive care unit (NICU), and 1 developed preeclampsia at 30 weeks of gestation with caesarean delivery and NICU admission. Of those 2 subjects with a history of prior preeclampsia, 1 developed preeclampsia at 30 weeks and delivered a premature infant while the other delivered a normal, full-term infant. These results show that early CPAP application were insufficient to prevent the occurrence of preeclampsia, spontaneous abortion, or premature birth in subjects with obesity and prior preeclampsia. However, none of the chronic hypertension subjects ultimately developed preeclampsia [54]. Subsequently, based on the findings of their first study, Poyares et al reported a second small randomized trial of CPAP versus no CPAP in 16 pregnant women with chronic hypertension and snoring recognized during the first 6 weeks of pregnancy. In this study, 7 pregnant women receiving CPAP experienced a drop in blood pressure as compared to an increase in blood pressure in 9 pregnant women in the control group [55]. Marked increases in blood pressure within the control group led to escalation of alpha methyldopa dosage, whereas in the CPAP treatment group blood pressure remained controlled without medication changes. None of the women in the CPAP group developed preeclampsia, while one of the control subjects did with resulting termination of the pregnancy at 33 weeks of gestation. All CPAP-treated patients experienced uncomplicated pregnancies and delivered infants with higher APGAR scores and significantly fewer infant visits during the postpartum period than controls. The apnea-hypopnea index documented by polysomnography in the CPAP-treated group was less than 5 events/hour in all subjects (mean3.1 \pm 1 events per hour) with an average minimum oxygen saturation of $92 \pm 1 \%$ with treatment [55]. Although limited by small sample size, these two studies present an impetus to further investigate the role of early CPAP intervention to prevent, lessen the severity, or delay the onset of preeclampsia in high-risk pregnant women. The potential benefits of prolonging gestation in utero are valuable in relation to infant growth and development, maternal safety, and hospital costs $[54,55]$. Larger-scale studies should be performed to demonstrate the potential benefit of CPAP intervention, considering the minimal side effects of CPAP largely consisting of nasal dryness and mask discomfort.

\section{TREATMENT OF OSA DURING PREGNANCY}

Guilleminault et al also reported successful CPAP treatment in pregnant women with diagnoses of SDB. Nasal CPAP was well-tolerated with full compliance, no hazard to infant outcome, and improvement in complaints of excessive daytime sleepiness and fatigue. From this study, some women complained of nasal congestion and had inferior turbinate engorgement related to pregnancy, but this was alleviated with humidification and mask adjustment. At 6 months, the CPAP pressures were increased in some subjects [56]. In addition, there have been case reports of CPAP treatment in women with obstructive sleep apnea during pregnancy. Lewis et al described a case of an obese pregnant women with OSA who subsequently developed pulmonary hypertension and severe anasarca at 29 weeks of gestation. She was treated with nasal CPAP during sleep and responded well, as evidenced by the massive diuresis that quickly followed, and she ultimately delivered at term via cesarean section due to transverse lie of the infant [57]. Another case report of a twin pregnancy involving severe obstructive sleep apnea was described by Langner et al In this instance, a 36-year-old woman carrying twins presented with complaints of snoring and nocturnal oxygen desaturations at 28 weeks of gestation. Polysomnography confirmed severe obstructive sleep apnea (AHI $=104$ events/hour, oxygen saturation nadir of $75 \%$ ). Treatment of OSA with nasal CPAP and supplemental oxygen followed by bilevel positive airway pressure set to "spontaneous mode was inadequate. When the bilevel was set on timed mode (i.e., with the bilevel functioning as a ventilator), there was a partial improvement of the sleep-disordered breathing (AHI= 32 events/hour, oxygen saturation nadir of $85 \%$ ). Because of the persistence of symptoms of preeclampsia despite therapy, a cesarean section was performed at 31 weeks of gestation with the birth of 2 healthy neonates. In this case preeclampsia was observed and a premature delivery could not be prevented. There were two unique elements that differ from the other cases: the onset of treatment was late (at 29 weeks of gestation) and it was a twin pregnancy [58]. Finally, Noseda reported on a morbidly obese, excessively sleepy woman with prior preeclampsia who was treated with 
nasal CPAP at 30 years of age. With nasal CPAP treatment, the patient had 3 completely normal pregnancies [59].

As nasal CPAP has been shown to be a safe and effective treatment of SDB during pregnancy, with only the need for regular follow-up to adjust the pressure during late pregnancy, this treatment approach should be discussed with pregnant women diagnosed with SDB. This is especially important given the exacerbation of SDB during pregnancy and the potential for adverse effects on pregnancy and fetal growth [56]. During the postpartum period, a re-evaluation of the severity of the syndrome with adjustment of CPAP pressure should occur considering the reports of improvement in SDB post-delivery [20].

\section{SUMMARY}

There are now accumulating data suggesting that not only OSA but SDB in general are independent risk factors for gestational hypertension and possibly preeclampsia, contributing to poor maternal and fetal outcomes such as low birth weight, small size for gestational age, and pre-term delivery. The interactions between preeclampsia and SDB are complex and bidirectional. While SDB can occur in association with the many physiological and hormonal changes of pregnancy, it also has a key role in blood control in preeclampsia, with obstructive respiratory events worsening the condition. The prevalence of SDB in gestational hypertension and preeclampsia is high. Interestingly, repetitive flow limitation as seen in upper airway resistance syndrome may be as detrimental as frank OSA. In any of these conditions, nasal CPAP treatment, at least if initiated early in pregnancy, has shown to be of benefit.

There are to date promising data indicating that early treatment with CPAP might be beneficial in high-risk pregnant women, especially among those with chronic hypertension and snoring. Treatment can control blood pressure and prevent the occurrence of preeclampsia with the associated poor fetal outcomes. Unfortunately, large studies are lacking. Currently there is no standard guideline for screening pregnant women for SDB. It is clear that women with pre-existing obesity, chronic or recently developed gestational hypertension, and a prior history of preeclampsia - all of whom are usually considered as having a "high-risk pregnancy" - should be evaluated for the presence of SDB (and not only OSA). This evaluation must include polysomnography with a nasal cannula signal to evaluate for flow limitation during sleep. If the investigation is positive, early treatment with nasal CPAP should be strongly considered. Further research should include large-scale studies to investigate the impact of early nasal CPAP application in pregnant women with preeclampsia risk factors and sleep-disordered breathing.

\section{CONFLICT OF INTEREST}

The authors confirm that this article content has no conflicts of interest.

\section{ACKNOWLEDGEMENTS}

We thank Brandon R. Peters, MD for his help in editing the manuscript.

\section{REFERENCES}

[1] Wang ARS, Karunmanchi SA. Preeclampsia: the role of angiogenic factors in its pathogenesis. Physiology 2009; 24: 147-58.

[2] Sibai BM, Dekker G, Kupferminc M. Preeclampsia. Lancet 2005; 365: 785-99.

[3] Sibai BM. Diagnosis and management of gestational hypertension and preeclampsia. Obstet Gynecol 2003; 102: 1003-6.

[4] Roberts JM, Taylor RN, Musci TJ, et al. Preeclampsia: an endothelial cell disorder. Am J Obstet Gynecol 1989; 161: 1200-4.

[5] Alderman BW, Sperling RS, Daling JR. An epidemiological study of the immunogenetic aetiology of preeclampsia. Br Med J 1986; 296: 372-4.

[6] Bhattacharya S, Campbell DM, Liston WA. Effect of body mass index on pregnancy outcomes in nulliparous women delivering singleton babies. BMI Public Health 2007; 7: 168.

[7] Lee KA, Zaffke ME, McEnany G. Parity and sleep patterns during and after pregnancy. Obstet Gynecol 2000; 95: 14-8.

[8] Driver HS, Shapiro CM. A longitudinal study of sleep stages in young women during pregnancy and postpartum. Sleep 1992;15: 449-53.

[9] Karacan I, Wayne H, Harman AW, et al. Characteristics of sleep patterns during late pregnancy and postpartum periods. Am J Obstet Gynecol 1968; 101: 579-86.

[10] Edwards N, Blyton DM, Kesby GJ, Wilcox I, Sullivan CE. Preeclampsia is associated with marked alterations in sleep architecture. Sleep 2000; 23: 9-25.

[11] Haeger M, Unander M, Andersson B, et al. Increased release of tumor necrotic factor-alpha and interleukin-6 in women with the syndrome of hemolysis, elevated liver enzyme, and low platelet count. Acta Obstet Gynecol Scand 1996; 75: 695-701.

[12] Jonsson Y, Ruber M, Matthiesen L, et al. Cytokine mapping of sera from women with preeclampsia and normal pregnancies. J Reprod Immunol 2006; 70: 83-91.

[13] Shoham S, Davenne D, Cady AB, Dinarello CA, Krueger JM. Recombinant tumor necrosis factor and interleukin 1 enhance slowwave sleep. Am J Physiol 1987; 253: R142-9.

[14] Edwards N, Middleton PG, Blyton DM, Sullivan CE. Sleep disordered breathing and pregnancy. Thorax 2002; 57: 555-8.

[15] Bobrowski R. Pulmonary physiology in pregnancy. Clin Obstet Gynecol 2010; 53: 285-300.

[16] Kambam JR, Handte RE, Brown WU, Smith BE. Effect of normal pre-eclamptic pregnancies on the oxyhemoglobin dissociation curve. Anesthesiology 1986; 65: 426-7.

[17] Loube DI, Poceta JS, Morales MC, Peacock MD, Mitler MM. Selfreported snoring in pregnancy: association with fetal outcome. Chest 1996; 109: 885-9.

[18] Franklin KA, Holmgren PA, Jonsson F, Poromaa N, Stenlund H, Svanborg E. Snoring, pregnancy-induced hypertension and growth retardation of the fetus. Chest 2000; 117: 137-41.

[19] Izci B, Vennelle M, Liston WA, Dundas KC, Calder AA, Douglas NJ. Sleep-disordered breathing and upper airway size in pregnancy and post-partum. Eur Respir J 2006; 27: 321-7.

[20] Edwards N, Blyton DM, Hennessy A, Sullivan CE. Severity of sleep-disordered breathing improves following parturition. Sleep 2005; 28: 737-41.

[21] Izci B, Riha RL, Martin SE, et al. The upper airway in pregnancy and preeclampsia. Am J Respir Crit Care Med 2003; 167:137-40.

[22] Bende M, Gredmark T. Nasal stuffiness during pregnancy. Laryngoscope 1999; 109: 1108-10.

[23] Ellegard EK. Pregnancy rhinitis. Immunol Allergy Clin North Am 2006; 26: 119-35.

[24] Lekas MD. Rhinitis during pregnancy and rhinitis medicamentosa. Otolaryngol Head Neck Surg 1992; 107: 845-8.

[25] Mabry RL. Rhinitis of pregnancy. South Med J 1986; 79: 965-71.

[26] Shushan S, Sadan O, Lurie S, Evron S, Golan A, Roth Y. Pregnancy-associated rhinitis. Am J Perinatol 2006; 23: 431-3.

[27] Sahin FK, Koken G, Cosar E, et al. Obstructive sleep apnea in preangncy and fetal outcome. Int J Gynecol Obstet 2008; 100: 1416.

[28] Chen YH, Kang JH, Lin CC, et al. Obstructive sleep apnea and the risk of adverse pregnancy outcomes. Am J Obstet Gynecol 2011; 206(2): 136.e1-5.

[29] Bourjelly G, Raker CA, Challhoub M, Miller MA. Pregnancy and fetal outcomes of symptoms of sleep-disordered breathing. Eur Respir J 2010; 36: 849-55. 
[30] Higgins N, Leong E, Park CS, et al. The Berlin questionnaire for assessment of sleep disordered breathing risk in parturients and non-pregnant women. Int J Obstet Anesth 2011; 20: 22-5.

[31] Olivarez SA, Maheshwari B, McCarthy M, et al. Prospective trial on obstructive sleep apnea in pregnancy and fetal heart rate monitoring. Obstetrics 2010; 202: 552-7.

[32] Izci B, Martin SE, Dundas KC, et al. Sleep complaints: snoring and daytime sleepiness in pregnant and pre-eclamptic women. Sleep Med 2005; 6: 163-9.

[33] Pien GW, Fife D, Pack AI, Nkwuo E, Schwab RJ. Changes in symptoms of sleep-disordered breathing during pregnancy. Sleep 2005; 28: 1299-305.

[34] Blyton DM, Sullivan CE, Edwards N. Reduced nocturnal cardiac output associated with preeclampsia is minimized with the use of nocturnal nasal CPAP. Sleep 2004; 27: 79-84.

[35] Redman CW, Beilin LJ, Bonnar J. Reversed diurnal blood pressure rhythm in hypertensive pregnancies. Clin Sci Mol Med 1976; Suppl. 3: 687-9.

[36] Beilin LJ, Deacon J, Michael CA, et al. Diurnal rhythms of blood pressure,plasma renin activity, angiotensin II and catecholamines in normotensive and hypertensive pregnancies. Clin Exp Hypertens 1983; 2(2): 271-93.

[37] Imai Y, Abe K, Munakata M, et al. Circadian blood pressure variations under different pathophysiological conditions. J Hypertens Suppl 1990; 8(7): S125-32.

[38] Hermida RC, Ayala DE, Iglesia M. Predictable blood pressure variability in healthy and complicated pregnancy. Hypertension 2001; 38: 736-41.

[39] Hermida RC, Ayala DE, Mojon A, et al. Blood pressure patterns in normal pregnancy, gestational hypertension and preeclampsia. Hypertension 2000; 36: 149-58.

[40] Hermida RC, Ayala DE, Iglesia M. Differences in circadian pattern of ambulatory pulse pressure between healthy and complicated pregnancies. Hypertension 2004; 44: 316-21.

[41] Edwards N, Blyton DM, Kirjavainen T, Kesby GJ, Sullivan CE. Nasal continuous positive airway pressure reduces sleep-induced blood- pressure increments in preeclampsia. Am J Respir Crit Care Med 2000; 162: 252-7.

[42] Edwards N, Blyton DM, Kirjavainen TT, Sullivan CE. Hemodynamic response to obstructive respiratory events during sleep are augmented in women with preeclampsia. Am J Hypertens 2001; 14: 190-5.

[43] Connolly G, Razak ARA, Hayanga A, Russell A, McKenna P, McNicholas WT. Inspiratory flow limitation during sleep in preeclampsia: comparison with normal pregnant and nonpregnant women. Eur Respir J 2001; 18: 672-6.

[44] Iber C, Ancoli-IsraelÂ S, Chesson A, Quan SF for the American Academy of Sleep Medicine. The AASM the Manual for scoring of sleep and associated events: Rules, Terminology and Techinical Specification. $1^{\text {st }}$ ed. Westchester Illinois: American Academy of Sleep Medicine 2007.

[45] Bao G, Guilleminault C. Upper airway resistance syndrome-one decade later. Curr Opin Pulm Med 2004; 10: 461-7.

[46] Reid J, Skomro R, Cotton D, et al. Pregnant women with gestational hypertension may have a high frequency of sleep disordered breathing. Sleep 2011; 34: 1033-8.

[47] Champagne K, Schwartzman K, Opatrny L, et al. Obstructive sleep apnea and its association with gestational hypertension. Eur Respir J 2009; 33: 559-65.

[48] Guilleminault C, Stoohs R, Shiomi T, Kushida C, Schnittger I. Upper airway resistance syndrome, nocturnal blood pressure monitoring and borderline hypertension. Chest 1996; 109: 901-8.

[49] Iczi-Balserak B, Pien GW. Sleep-disordered breathing and pregnancy: potential mechanisms and evidence for maternal and fetal morbidity. Curr Opin Pulm Med 2010; 16: 574-82.

[50] Baumwell S, Karumanchi SA. Preeclampsia: clinical manifestation and molecular mechanisms. Nephron Clin Pract 2007; 106: c72-81.

[51] Jerath R, Barnes VA, Fadel HE. Mechanism of development of preeclampsia linking breathing disorders to endothelial dysfunction. Med Hypotheses 2009; 73: 163-6.

[52] Bachour A, Teramo K, Hiilesmaa V, Maasilta P. Increased plasma levels of inflammatory markers and upper airway resistance during sleep in preeclampsia. Sleep Med 2008; 9: 667-74.

[53] Yinon D, Lowenstein L, Suraya S, et al. Preeclampsia is associated with sleep-disordered breathing and endothelial dysfunction. Eur Respir J 2006; 27: 328-33.

[54] Guilleminault C, Palombini L, Poyares D, et al. Preeclampsia and nasal CPAP: part 1 Early intervention with nasal CPAP in pregnant women with risk factors for preeclampsia: preliminary findings. Sleep Med 2007; 9: 9-14.

[55] Poyares D, Guilleminault C, Hachul H, et al. Preeclampsia and nasal CPAP: part 2. Hypertension during pregnancy, chronic snoring and early nasal CPAP intervention. Sleep Med 2007; 9: 1521.

[56] Guilleminault C, Kreutzer M, Chang JL. Pregnancy, sleep disordered breathing and treatment with nasal continuous positive airway pressure. Sleep Med 2004; 5: 43-51.

[57] Lewis DF, Chesson AL, Edwards MS, Weeks JW, Adair CD. Obstructive sleep apnea during pregnancy resulting in pulmonary hypertension. South Med J 1998; 91: 761-2.

[58] Langner S, Halank M, Kolditz M, Schiemanck S, Hoffken G. Twin pregnancy and severe obstructive sleep apnea. Z Geburtsshilfe Neonatol 2007; 211: 93-7.

[59] Noseda A, Linkowski P. Three pregnancies on nasal CPAP: a case report. Rev Med Brux 2007; 28: 191-3.

\author{
$\overline{\text { Received: January 01, } 2012 \quad \text { Revised: September 06, 2012 Accepted: January 26, 2013 }}$ \\ (C) Tantrakul et al.; Licensee Bentham Open.
}

This is an open access article licensed under the terms of the Creative Commons Attribution Non-Commercial License (http://creativecommons.org/licenses/by$\mathrm{nc} / 3.0 /$ ), which permits unrestricted, non-commercial use, distribution and reproduction in any medium, provided the work is properly cited. 